\title{
Adapt!Evaluation - Evaluation for Design and Re-Design of Production Systems based on Axiomatic Design
}

\author{
Markus Stäbler ${ }^{1, *}$, Jakob Weber ${ }^{1}$, and Kristin Paetzold ${ }^{2}$ \\ ${ }^{1}$ Daimler AG, Process Development and Materials, 71059 Sindelfingen, Germany \\ ${ }^{2}$ Universität der Bundeswehr, Institut für Technische Produktentwicklung, 85577 Neubiberg, Germany
}

\begin{abstract}
Since trends of our society are changing, the automotive industry has to handle new requirements within their production like vague quantities of production, fast integration of new technologies and an increasing variety of diversity. Therefore conventional life-cycles of production systems are broken up and re-design and changeability of complex systems have to be considered during their whole life time. Within this framework the paper proposes a novel approach to enable change during the life cycle of a production system and to enhance the usability of a re-design and change process by consistent interaction between the design and evaluation method. Thereby the focus is on the linkage of design and evaluation process to pursue a decrease of complexity and to point out the benefits which can be found by using Axiomatic Design Theory in this holistic context of the production system life-cycle. To validate the results of the paper and show up their benefits, a use case will describe and discuss the research.
\end{abstract}

\section{Introduction}

Due to shifting trends of our society, such as globalisation, individualisation and our fast moving nature, production systems are faced by huge challenges like vague quantities of production, fast integration of new technologies and an increasing variety of diversity. Since the conventional production system life-cycle is stretched to its limit, a new life-cycle with integrated phases of change or re-design is striven for (Fig. 1). To provide a good foundation the paper proposes a novel approach by combining the approach by Weber based on Axiomatic Design Theory (AD) with the evaluation approach for Changeability by Staebler. Therefor the holistic method Adapt! will be provided first to design a changeable production system and secondly, during its lifetime, to detect where and how much the production system has to be changed or to be re-designed to respond to new requirements. Thereby the focus of this paper is on the linkage of these two approaches and the benefit what may result.

In the first part of the paper the state of the art will be discussed and some basics will be named. Based on that, the new approach will be introduced. Moreover to explain the functionality of the method and validate the results, a use case of the automotive industry will be shown and discussed.
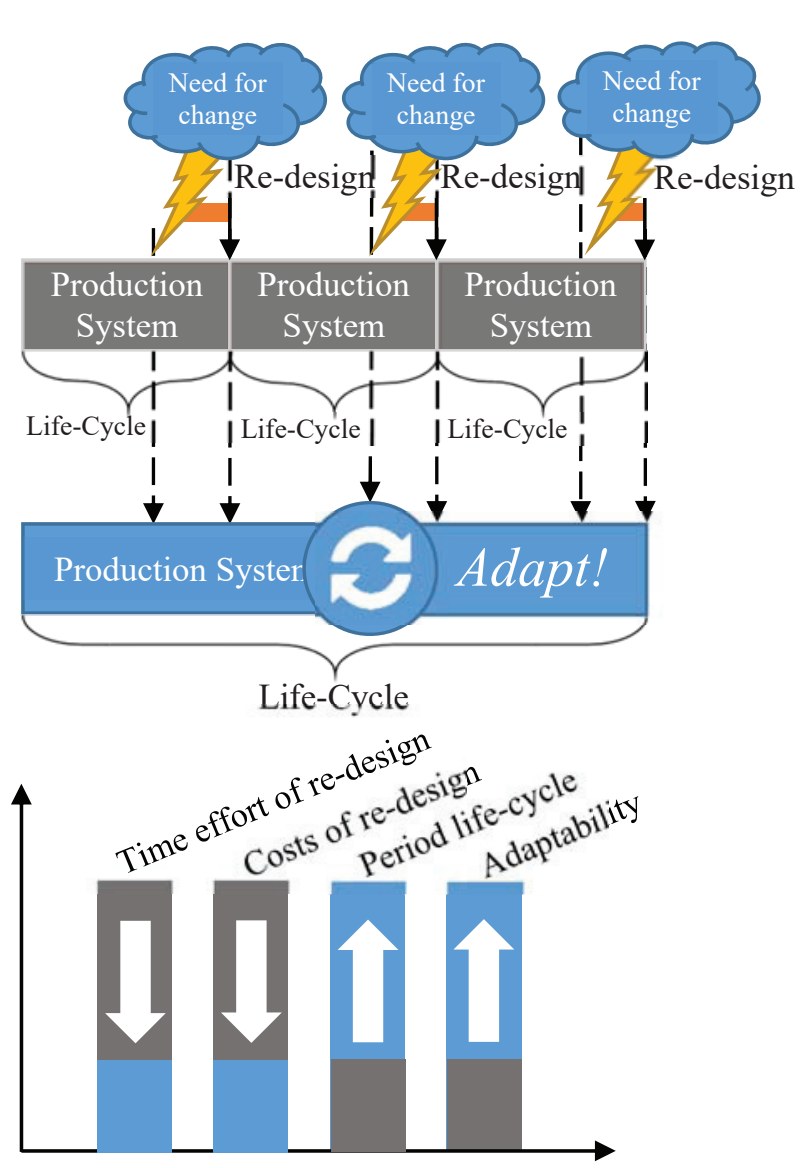

Fig. 1. Method Adapt! to ensure a long and adaptable lifecycle.

\footnotetext{
* Corresponding author: markus.m.staebler@daimler.com
} 
Three key questions concerning the paper can be formulated:

- Is AD a suitable design method in a volatile environment?

- How can a holistic approach (Initial Design, Evaluation during life-cycle and Re-Design) in the context of changeability and based on AD look like?

- What are the benefits to build up an evaluation process upon a design process based on $\mathrm{AD}$ ?

\section{State of the art}

To get a simultaneous knowledge base, in this chapter some basics, like changeability and life cycle in the context of production systems, will be explained. Further the principles of Axiomatic Design and related works dealing with re-design will be summarized.

\subsection{Production system}

According to Nyhuis a production system is a sociotechnical system which converts some kind of input through value-adding and associated processes into output values [1]. This paper focuses on production system as a mechanical system which can be consist of a single assembly station up to a complete assembly shop.

\subsubsection{Life cycle of production systems}

The term lifecycle can be defined as the "development of an organism in its various stages from initiation through maturity" [2]. This definition can be adopted to the topic product life-cycle or production life-cycle which are of interest for this approach (Fig. 2). A sizeable amount of literature exists on product lifecycle management.

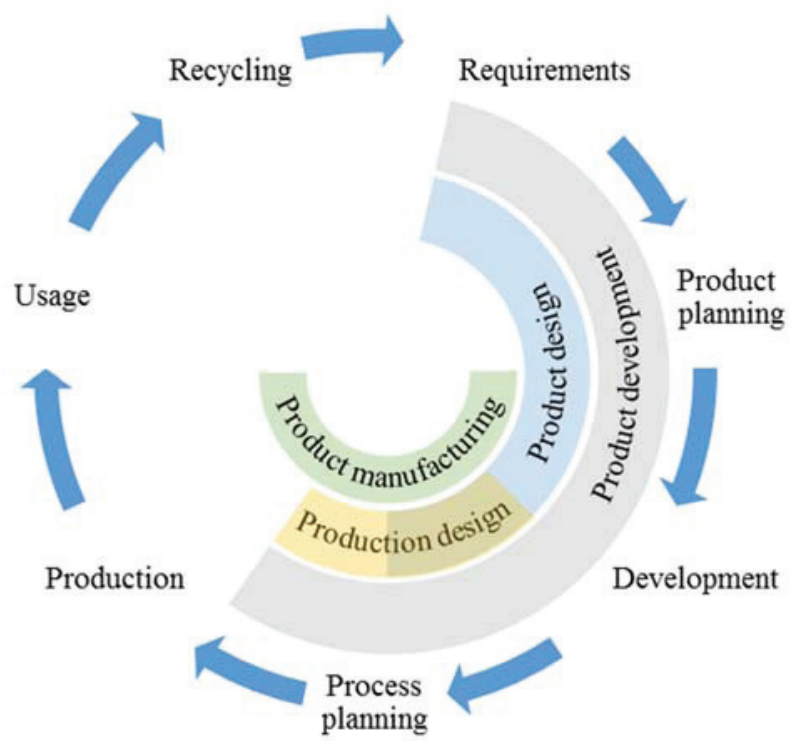

Fig. 2. life-cycle based on [3].

It is noticeable that the focus of previous research is not on change or re-design of production systems during one life-cycle $[3,5,4]$.

\subsubsection{Changeability}

"Changeability has been proposed as an umbrella concept that encompasses many aspects of change on many levels within the manufacturing enterprise" $[6,7]$. In this context, changeability of a production system is seen as a shift of an intrinsic flexibility corridor (Fig. 3) $[1,8]$.

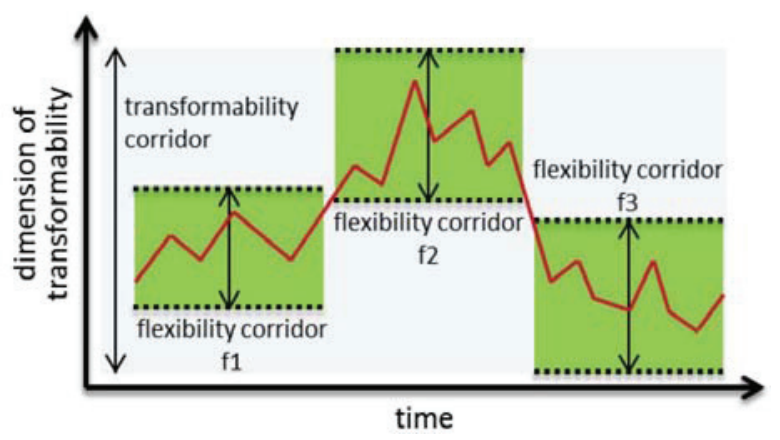

Fig. 3. Changeability as a shift of flexibility [9] based on [8].

To favour a production system to be changeable, several change enablers can be named [7]. Wiendahl et al. distinguish between three levels of a changeable physical system - First the Reconfigurable Manufacturing System, second the Reconfigurable Assembly System and third the Transformable Factory. Thereby the change enablers modularity and scalability serve as an intersection of the named physical system layers. [6]

\subsection{Reducing complexity}

Related to Pahl\&Beitz, technical systems, like production systems, tend to be complex systems with a high grade of dependencies [10]. To cope well with complexity during the design process some kind of structure is needed [11]. One of the main characteristics of the Axiomatic Design Theory is structuring and reducing complexity [12, 13]. Several more design methods have their focus on structuring and reducing complexity $[10,11]$, but at this point it is not meaningful to mention them in-depth.

\subsection{Axiomatic Design}

Axiomatic Design, developed by Suh, is a design method based on two axioms: first the Independence Axiom which aims to avoid dependencies between design parameters and functional requirements and secondly the Information Axiom in order to choose the right solution with less complexity and as few information content as possible $[12,14]$. The theory consists of four domains $[12,15]$. First of all, Stakeholder Requirements (SR), has to be defined. Then Functional Requirements (FR) has its focus on "what" function should be realized and the Design Parameters (DP) contains the information "how" you want to achieve the corresponding FR. As a supporting design tool hierarchical tree and Design Structure Matrix are used. Within the hierarchical tree 
requirements and DP are fragmented for solution finding and, by using Design Structure Matrix (DSM), dependencies will be shown.

\subsubsection{Axiomatic Design in context of changeability and re-design}

Meanwhile there is a very extensive literature on Axiomatic Design in context of changeability and redesign. It would go beyond the constraints of this paper mentioning all of them. Hence, this paper makes a selection to give the reader a rough overview about the state of the art.

Farid developed a measurement possibility for reconfigurability based on $\mathrm{AD}$ and widen the topic with some more change enablers like integrability or convertibility [16]. In the next step the author combined his results with a model based system engineering [15]. As a case study for re-design Gabriel-Santos et al. can be named. The focus of his approach is on Axiom 1 to increase productivity of the manufacturing system [17]. Holzner et al. combining FR with requirements for change to achieve a flexible and changeable manufacturing system, focusing on small and mediumsized enterprises.

\subsubsection{Approach by Weber et al.}

Weber et al. modified AD by focusing on Axiom 1 . Thereby they want to achieve a simplification to increase the usability and acceptance in the industry (Fig. 4) [18].
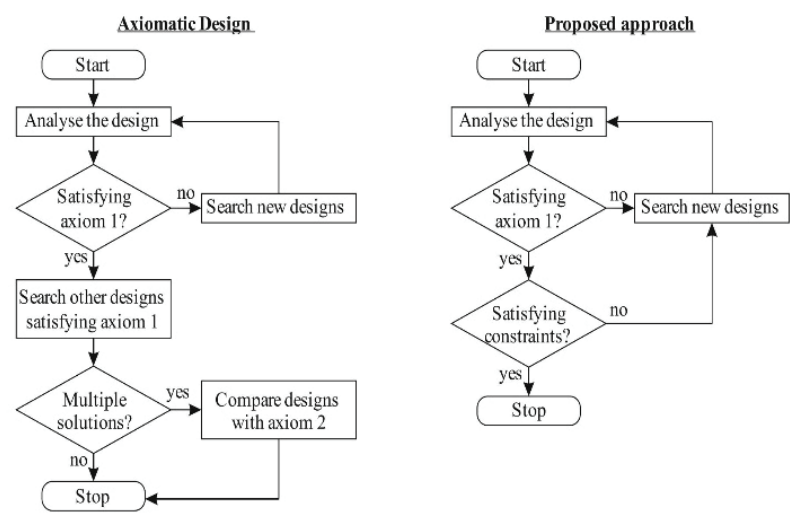

Fig. 4. Modification of AD to increase acceptance [18].

Aware of that, by neglecting Axiom 2, the optimum might not be determined, the results are good and robust enough by considering the dependencies of the system. As a second adaption colour-coding was established [19]. By colouring the Design Structure Matrix, it is possible to see the FRs' origins. So it can serve as a tool to enhance discussions with the company or the concerned division of the company.

\section{Theoretical consideration}

In order to achieve a changeable production system the procedure of Weber et al. is used which is based on AD Theory. Due to volatile circumstances the designed production system is exposed to a variety of change requirements during its lifecycle. Therefore, in this approach the method is extended with the evaluation approach by Staebler et al. to generate a holistic approach for Design and Re-Design (Fig. 5). The evaluation method answers the questions: "What subsystem should be changed first" and "What is the right degree of changeability" within the production system. However the focus of this paper is not about how the method works in general but if it has an added value by combining these two approaches and if $\mathrm{AD}$ is appropriate for this holistic approach.

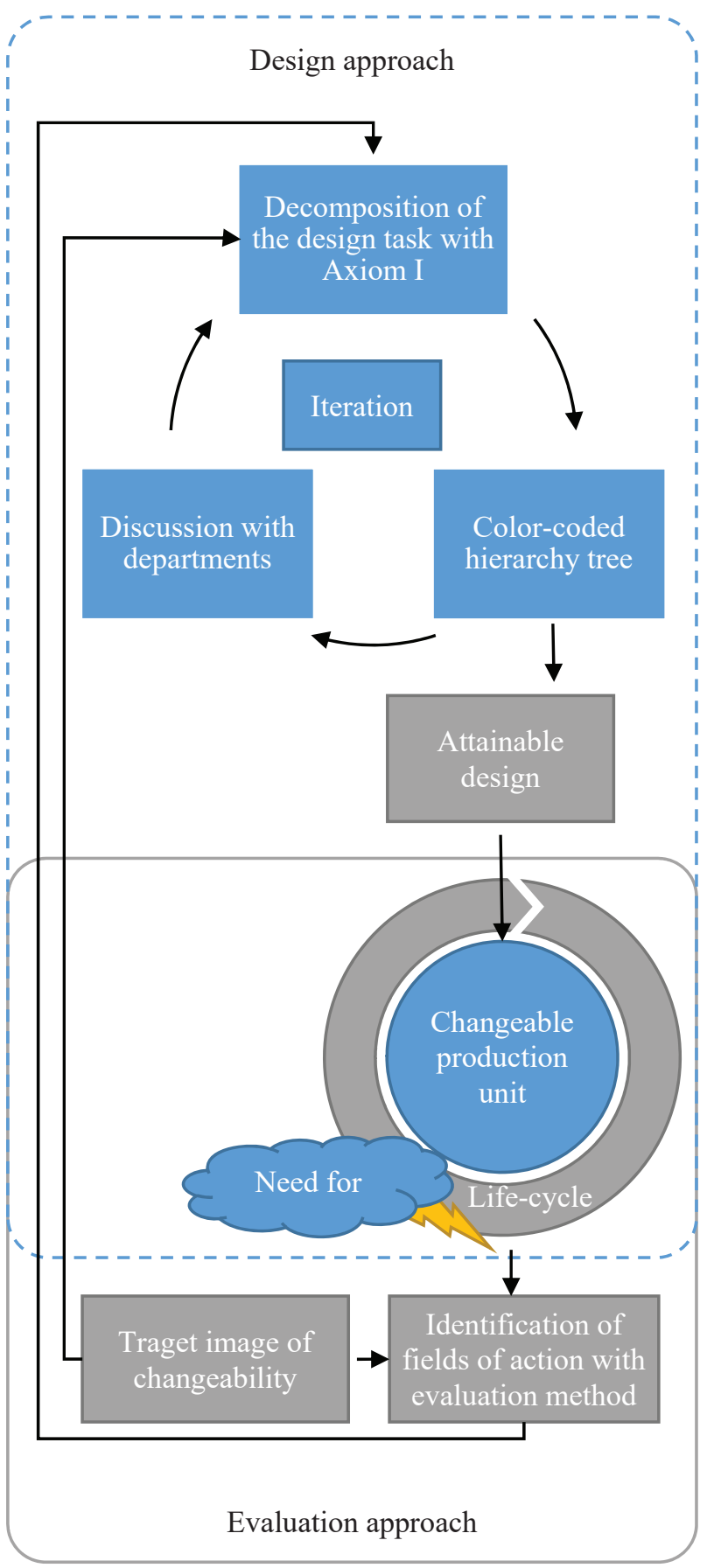


Fig. 5. Linkage between design and evaluation approaches.

So first the linkage between these two approaches will be discussed in the following subchapters. 


\subsection{Evaluation process}

The method has three cornerstones on which the evaluation is funded: the effort (when you want to implement change in a system), the effectivity (how positive is the effect of the entire system in context of changeability (when you change a part of it) and the dependencies within a system. As a first step subsystems have to be defined. Subsystems can be generated because of company structure, organisational reasons or by design parameters. To reduce complexity of the change process, subsystems should have as few dependencies as possible. Another reason can be named because neutrality favours changeability of a system. [1, 20]

\subsubsection{Dependencies as a main evaluation parameter}

One fundamental principle of the evaluation method is based on the Pareto Principle. That means that it might be possible to achieve $80 \%$ of the results with $20 \%$ effort. So under the assumption that independent subsystems are easier to change, the idea is to consider first subsystems which are as independent as possible. In reality a $100 \%$ independence of entire systems is of course not possible. That is why an evaluation of dependencies within a system has to be made.

\subsubsection{Required input variables}

To sum up, the evaluation method needs, in the context of dependencies, the information which subsystems are suitable for the evaluation and the following change process. Thereby the subsystems should have as few dependencies as possible and the degree of dependence should be determined.

\subsection{Results of the design process}

By using the $\mathrm{AD}$ theory in this case the primary goal is to achieve a changeable production system. During the design process a hierarchy tree and a Design Structure Matrix will be generated which enables to accomplish the primary goal. Sometimes it is seen as a means to an end, but in our case it implies a lot of information which can be used as required input variables of the following evaluation process.

\subsubsection{Derived information from hierarchy tree}

To avoid unnecessary work and complexity of the evaluation process, it is important to choose the right system layer. In practice it has been shown that the hierarchy tree is a good tool to get a fast overview over the complete production system and its degree of complexity. To get a first impression of the entire system it might be useful to enclose only the first layers of the hierarchy tree to generate subsystems. Then the results are getting more on an abstract level. Secondly, some branches of the tree can possibly be neglected. This leads to a reduction of evaluation time. Through its clear structure, the biggest benefit of the Hierarchy tree is the derivation of subsystems (Fig.6). To get a fast overview about defined subsystems each will be marked with a different colour. Subsystems can consist of one or more DPs on the same or different layers. By complex systems an iteration process with the DSM may be useful. By handling complex systems planning teams need a basis for their discussion and validation of decisions. So with this tool decisions get more transparent.

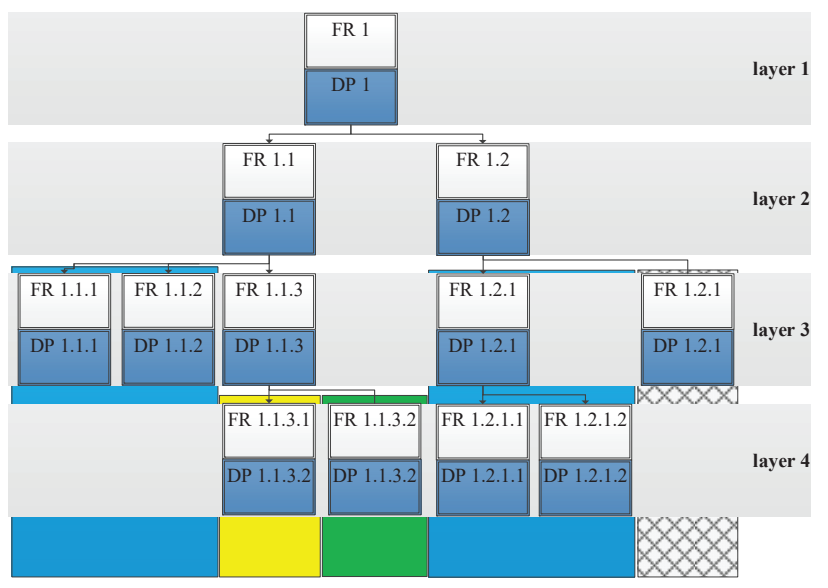

Fig. 6. Deriving subsystems based on hierarchy tree.

\subsubsection{Derived information from DSM}

As mentioned before, during the evaluation process the Design Structure Matrix is a tool to generate a changeable production system. In addition, it helps to quantify dependencies between subsystems. By deriving detected subsystems into the Matrix, dependencies can be seen very obviously (Fig. 7). Thereby FR $\mathrm{i}$ to DP $\mathrm{i}$ will be seen as independent and marked with green, dependencies within the same layer will be highlighted with yellow and dependencies to other layers with red. Intrinsic dependencies of a subsystem can be neglected and only relations between subsystems are of interest to an upcoming evaluation process.

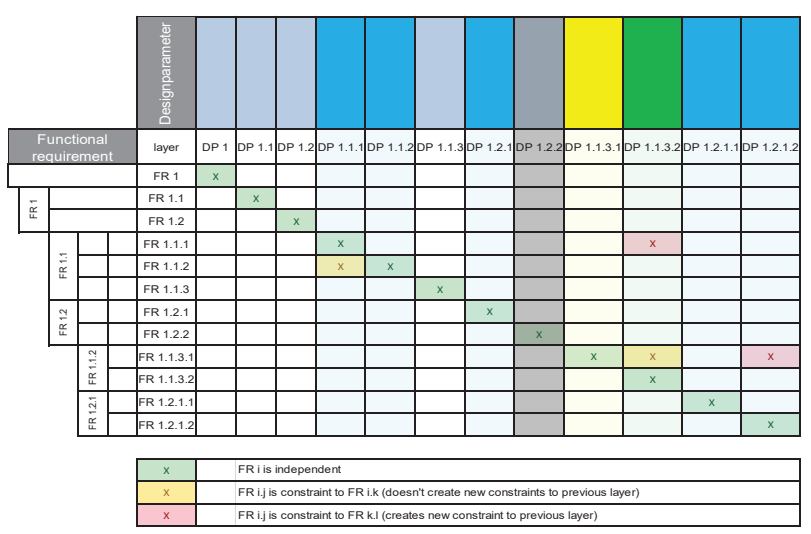

Fig. 7. Quantification of dependencies.

Because of searching for subsystems with as few dependencies as possible, it will not be distinguished 
between positive and negative dependencies. Indeed it will be differentiated between dependencies within the same layer or with other layers. According to the latest research, dependencies within a layer will be rated as one and dependencies to other layers as two. But this scale of assessment has to be confirmed by further use cases and research.

\section{Use case}

To improve changeability of the entire assembly production system in the automotive industry, a new concept of an entire assembly production system should be invented. Therefore the status quo of a conveyor system, three production units and the logistic system was recorded by means of Axiomatic Design. Optimally the design process was already realized by using Axiomatic Design. In this case the production system is a result of several decades of iteration. But to validate the research it is also sufficient to use $\mathrm{AD}$ afterwards. Derived from $\mathrm{AD}$, the demand for action in the context of changeability was determined. Figure 8 shows the results of the status quo visualized by a hierarchical tree.

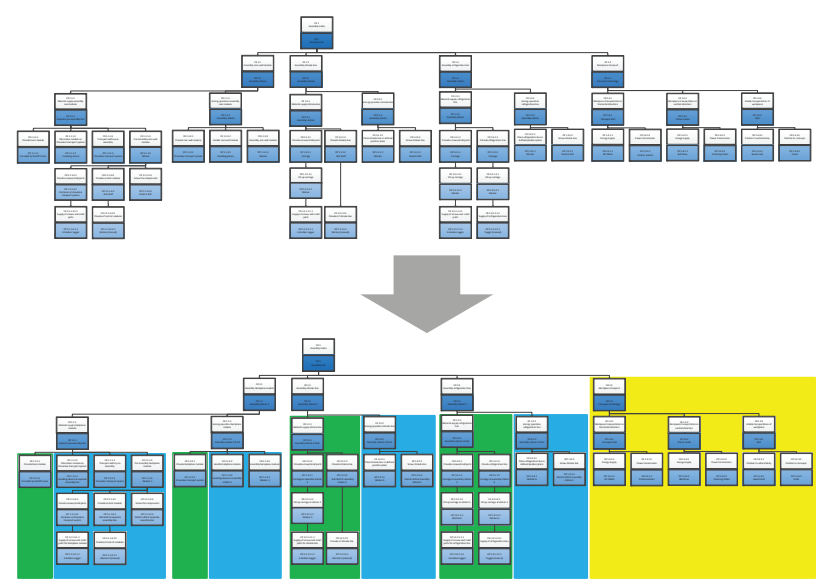

Fig. 8. Use case - building subsystems.

Based on this, three subsystems were built and visualized by colour. The yellow marked subsystem shows the conveyor system, blue all assembly operations and green all objects regarding logistic. Following subsystems were transferred to the dependency matrix (Fig. 9). Thereby all layers below the marked subsystems were considered too. After determining the dependencies, it can be stated that the conveyor system has less dependencies (1) followed by the subsystem assembly operations (11). The subsystem logistic has most dependencies (36). So by neglecting the other two cornerstones of the evaluation method, it can be deduced that we should first start to have the focus on the subsystem conveyor system because of its encapsulated structure.

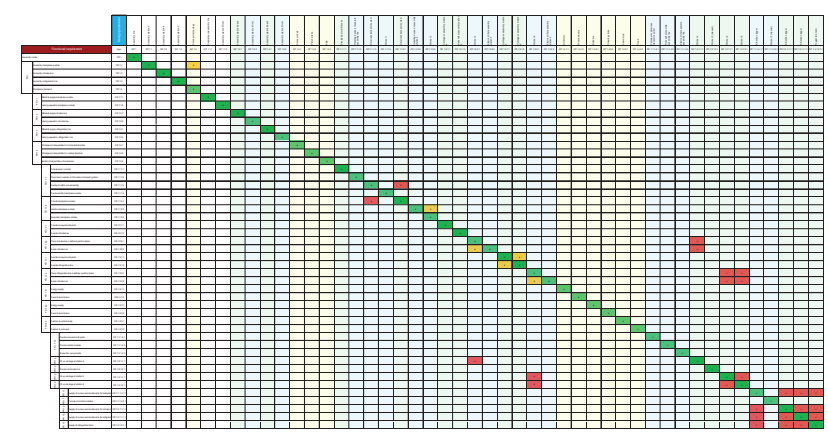

Fig. 9. Use case - quantification of dependencies.

\section{Discussion}

Regarding the introduced approach, results of the use case will be discussed and further key questions of the paper will be answered. The use case showed an improvement of the usability of the entire method compared to former development. Usability was measured by the effort of time during evaluation. Reduction of costs by implementing a continuous process of change and re-design in the life-cycle of a production unit can only be estimated and referred to existing literature about the benefit of intrinsic changeability of a system. Hence by increasing the userfriendliness the acceptance of using a method during the lifecycle becomes visible. Thus it can be stated that AD is a suitable design method in a volatile environment. As already mentioned there are numerous benefits to build up an evaluation process upon a design process with Axiomatic Design. Based on the independence, axiom suitable subsystems could be generated. It appears from the use cases that the complexity by determining dependencies is reduced immensely which leads to fast results.

\section{Conclusion and outlook}

This paper presented a novel approach by combining a modified axiomatic Design approach with a evaluation method for determining fields of action to increase the changeability of a production system and give answers to a continuously re-design process. It was shown that the method Adapt! improves the applicability of evaluation method based on axiomatic design by a lean linkage. After explaining the novel method the linkage of AD and the evaluation process will be demonstrated by an use case. In following research the method Adapt! has to be validated by more use cases. Furthermore it should be applied during a whole lifecycle of a product to manifest the benefits of it. 


\section{References}

1. P. Nyhuis, Prod. Zent., Changeable Production Systems: Shaping Today the Industry of Tomorrow (in German), 166, (2008)

2. H. Herrmann, H. Bucksch, Dict. Geotech. Engineering, 56 (2014)

3. M. Eigner, R. Stelzer, Springer, Product Lifecycle Management: A Guideline for Product Development and Life Cycle Management (in German), 2, 434 (2013)

4. A. Saaksvuori, A. Immonen, Springer, Product Lifecycle Management, 3, 257 (2008)

5. J. Stark, Springer, Product Lifecycle Management: 21st Century Paradigm for Product Realisation, 573 (2011)

6. H.-P. Wiendahl, H.A. ElMaraghy, P. Nyhuis, M.F. Zäh, H.-H. Wiendahl, N. Duffie, M. Brieke, CIRP Manuf. Techn., Changeable Manufacturing Classification, Design and Operation, 56 783-809 (2007)

7. H.A. ElMaraghy, Springer, Changeable and Reconfigurable Manufacturing Systems, 414 (2009)

8. M.F. Zäh, N. Möller, W. Vogl,, Proceed. Of CARV, Symbiosis of Changeable and Virtual ProductionTheEmperor's New Clothes or Key Factor for Future Success? (2005)

9. M. Stäbler, J. Weber, K. Paetzold, Proceed. Of CIRP CATS, Implementing Changeability in a System with Prioritization Indicators, 44, 50-54 (2016)

10. G. Pahl, W. Beitz, J. Feldhusen, K.-H. Grote, Springer, Engineering Design: A Systematic Approach (in German), 6, 780 (2005)

11. U. Lindemann, M. Maurer, T. Braun, Springer, Structural Complexity Management: An Approach for the Field of Product Design, 247 (2009)

12. N.P. Suh, Oxford Univ. Press, Axiomatic design: Advances and applications, (2001)

13. R. Fiege, Gabler, Axiomatic Design: A Method for Service-Oriented Modeling (in German), 1, 228 (2009)

14. N.P. Suh, Oxford Univ. Press, The principles of design, (1990)

15. A.M. Farid, N.P. Suh, Springer, Axiomatic design in large systems: Complex products, buildings and manufacturing systems, 322 (2016)

16. A.M. Farid, Proceed. of ICAD, Axiomatic design and design structure matrix measures for reconfigurability \& its key characteristics in automated manufacturing systems, (2014)

17. A. Gabriel-Santos, A.M. Gonçalves-Coelho, P. Santos, J. Fradinho, A. Mourão, Proceed. of CIRP ICAD, Redesigning a Manufacturing System Based on Functional Independence: The Case of a Tree Nursery, 34, 206-211 (2015)
18. J. Weber, J. Kößler, K. Paetzold, Proceed. of ICED, An approach for industrial application of axiomatic design. International conference on engineering design (2015)

19. J. Weber, D. Förster, J. Kößler, K. Paetzold, Proceed. of ICAD, Design of Changeable Production Units within the Automotive Sector with Axiomatic Design, 34, 93-97 (2015)

20. C.L. Heger, Prod. Zent., Evaluation of Changeability of Factory-Objects (in German), 224 (2007) 